\section{than others?}

\section{Tuominen $\mathbf{R}$ \\ Acta Odont Scand 200I; 59: 79-82}

\section{Mjör IA, Moorhead JE et al. Int Dent J 2000; 50: 36 I-366}

Secondary caries was the principal reason given for replacement.

In a Norwegian survey it was found that 243 general dental practitioners had placed 24,429 restorations, of which 22,931 were in permanent teeth, and of these, $49 \%$ were replacements. Reasons for replacement were recorded for 9,805 restorations ( $87 \%$ ); of these, 5,731 were amalgams, 2,952 were composites, and 380 were glass ionomers in adults $19 \mathrm{yrs}$ and older; in younger subjects, respective figures were 273,187 and 282.

In the older group, the main reasons for replacement were secondary caries (amalgam $57 \%$, composite $47 \%$, glass ionomers $50 \%)$ and restoration fracture $(25,24,23 \%)$. In the younger group, respective figures were 74,48 and 56\%, 12, 28 and $18 \%$.

Pain or sensitivity was rarely the reason for replacement. Discolouration more frequently led to composite replacement in the older subjects, and in the younger subjects, poor anatomical form was given as a reason for $13 \%$ of glass ionomer replacements. Female clinicians diagnosed secondary caries more often than males, and the youngest clinicians diagnosed it more frequently than the most experienced.

\section{Oral medicine}

\section{Effect of highly active antiretroviral therapy on frequency of oral warts

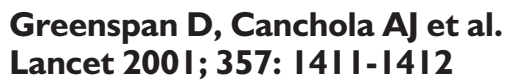

Whilst some lesions decreased with HAART, warts increased.

This study retrospectively examined records for $1280 \mathrm{HIV}$ positive subjects seen from 1990 to 1999 in an oral medicine clinic in San Francisco. Treatment which excluded protease inhibitors was defined as antiretroviral therapy (ART) and treatment including both antiretroviral agents and protease inhibitors was defined as highly active (HAART).

Over the 9 years, oral candidosis, hairy leucoplakia and Kaposi's sarcoma showed significant decreases, whereas oral warts and salivary gland disease increased, and aphthous ulcers showed no overall change. Before protease inhibitors were introduced, candidosis, leucoplakia and warts were not associated with ART use. Afterwards, candidosis incidence was lower in ART and HAART patients than others, but the prevalence of warts was higher in patients on ART and HAART.

The authors consider that the reduction in oral infection with HAART is not surprising, but the increase in oral warts is unexpected, and presents some difficulties in management, as they may be progressive, uncomfortable and unaesthetic with a tendency to recur.
Prospective patients prefer certain characteristics in dental advertisements.

Advertising is a necessary part of a market economy, although many professionals have resisted it. A standard market research technique was used in this study, to investigate the views of Finnish people on dentists' yellow page advertisements. In each of 5 cities, 20 men and 20 women were interviewed at random in each of 5 age groups from 20 yrs upwards.

A double-page spread of dentists' advertisements was chosen from the Helsinki directory, including a variety of 17 display ads and 24 conventional listings. Subjects were asked to choose the most and least attractive ads and give their reasons.

The 5 most attractive ads were significantly larger with less text density than others. Use of a heading, listing of services or specialties and a logo were all significantly associated with attractiveness, but use of frames was associated with unattractiveness. Subjects reported use of pictures, clarity and large size as reasons for attractiveness, and small print, boringness and muddled appearance for unattractiveness. All 3 ads designed by professional advertisers were among the 5 most attractive, and the 5 least attractive were by the dentists themselves.

Oral hygiene

How do routines of daily activities and flexibility of daily activities affect tooth-cleaning behavior?

\section{Abegg C, Croucher R et al. J Public Health Dent 2000; 60: I 54- 58}

A more flexible working day was associated with more effective tooth cleaning and healthier gingivae.

The flexibility of daily activities is a factor which may affect such behaviours as tooth cleaning. In a South American city, a total of 471 subjects was recruited in factories, offices, banks, shops and hospitals, a minimum of 50 for each of 8 groups stratified according to 3 divisions: men or women, high or low social class, and high or low flexibility of daily activities. Subjects assessed the extent to which their daily activities were flexible in terms of when they were performed, and also the extent to which regular daily activities formed a routine. Dental plaque and gingival bleeding were clinically assessed.

After adjustment for age, gender and marital and socioeconomic status, tooth-cleaning pattern and routine daily activities were significantly associated (odds ratio 2.3), but there was no association between routines and gingival bleeding levels. Flexibility of daily activities was significantly associated with tooth-cleaning frequency $(\mathrm{OR}=1.6), \mathrm{OH}$ performance (2.7), and gingival bleeding (2.3). The authors also recommend that patients should be instructed in the simplest appropriate methods of oral hygiene. 